5th International Workshop on Astronomy and

Relativistic Astrophysics (IWARA2011)

International Journal of Modern Physics: Conference Series

Vol. 18 (2012) 18-24

(C) World Scientific Publishing Company

DOI: $10.1142 / \mathrm{S} 2010194512008148$

\title{
HOLONOMY TRANSFORMATION IN THE LENSE-THIRRING SPACETIME
}

\author{
J. GOMES DE ASSIS \\ Departamento de Matemática, Universidade Federal da Paraíba \\ 58051-970 João Pessoa,Pb, Brazil \\ assis@mat.ufpb.br \\ V. B. BEZERRA \\ Departamento de Física, Universidade Federal da Paraíba, \\ 58051-970 João Pessoa, Pb, Brazil. \\ valdir@fisica.ufpb.br
}

\begin{abstract}
We compute the holonomy transformation for circles on the equatorial plane in the LenseThirring spacetime taking into account the appropriate modification of this quantity due to the rotation of the source. In parallel transporting vectors we examine how the rotation affects the holonomy transformation.
\end{abstract}

Keywords: Loop variable; Holonomy transformation; Lense-Thirring spacetime.

PACS numbers: 03.65.Ge, 14.80.Hv

\section{Introduction}

In the loop space formalism for gauge theories ${ }^{1}$ the fields don't depend on spacetime points, but on curves in the spacetime under consideration. The fundamental quantity that arises from this approach, the non-integrable phase factor ${ }^{2}$ (loop variable) contains more precise informations concerning an arbitrary gauge field than the field strength or the integral of the vector potential ${ }^{2}$.

The extension of the loop space formalism to the theory of gravity was first considered by Mandelstam ${ }^{3}$ who established several equations involving the loop variable which is a quantity related with the parallel transport of a vector along a curve. The loop variable for a closed curve is the so-called holonomy transformation.

In the framework of gravity, some calculations concerning the holonomy transformation have been done, as for example, the computation of the loop variable in the Kerr spacetime ${ }^{4}$. Among other calculations of this quantity, we can mention the one corresponding to a class of topological defects ${ }^{5}$ and others spacetimes ${ }^{6-15}$. One of the physical motivations to calculate the holonomy is the possibility to use this quantity to investigate the gravitomagnetism ${ }^{16}$ which can be considered as an analog of the gravitational Aharonov-Bohm effect ${ }^{17,18}$. 
Let us suppose that $v^{\alpha}$ is a vector at a point $P$ of a curve $C$ in a given spacetime. Thus, we can produce the vector $u^{\alpha}$ at $P$, which in general will be different from $v^{\alpha}$, by parallel transporting $v^{\alpha}$ around $C$. In this case, we associate with the point $P$ and the curve $C$ the linear map $U_{\beta}^{\alpha}$ such that $u^{\alpha}=U_{\beta}^{\alpha} v^{\beta}$. This map $U_{\beta}^{\alpha}$ is called the loop variable associated with the point $P$ and the curve $C$. In the particular case in which the curve is closed, this quantity is called holonomy transformation.

If we choose a tetrad frame and a parameter $\lambda \in[0,1]$ for the curve $C$ such that $C(0)=C(1)=P$, then in parallel transporting the vector $v^{\alpha}$ from $C(\lambda)$ to $C(\lambda+d \lambda)$, the component of this vector changes by $\delta v^{\alpha}=M_{\beta}^{\alpha}[x(\lambda)] v^{\beta} d \lambda$, where $M_{\beta}^{\alpha}$ is a linear map which depends on the tetrads, the affine connection of the spacetime and the value of $\lambda$. Then, the holonomy transformation $U_{\nu}^{\mu}$ is given by the ordered matrix product of the $N$ linear maps as

$$
U_{\alpha}^{\beta}=\lim _{N \rightarrow \infty} \prod_{i=1}^{N}\left[\delta_{\alpha}^{\beta}+\left.\frac{1}{N} M_{\alpha}^{\beta}[x(\lambda)]\right|_{\lambda=i / N}\right] .
$$

We can write expression given by Eq. (1) as

$$
U_{L}(C)=P \exp \left[-\int_{B}^{A} \Gamma_{\mu}(x(\lambda)) \frac{d x^{\mu}}{d \lambda}\right]
$$

to indicate the linear holonomy, where $\Gamma_{\mu}$ is the tetradic connection, $A$ and $B$ are the initial and final points, respectively, of the curve $C$ and $P$ means the ordered product along this curve.

Note that the holonomy given by eq.(2), which we will call linear holonomy, $U_{L}$, is not sufficient to distinguish spacetimes with or without rotation ${ }^{19}$ and therefore, in order to have the correct expression for the holonomy corresponding to a curve in a spacetime generated by a rotating body it is necessary also to consider the holonomy that is proportional to the angular moment of the source, which is called translational holonomy. In this case, we adopt the concept of curve development in a manifold ${ }^{20}$ to calculate this part of the holonomy. Thus, the total holonomy is obtained by summing up the contributions arising from the linear and translational holonomies.

Now, let us consider the contribution to the loop variable arising from the translation part. If we have rotation, it will give a contribution to the total holonomy due to the rotation, through the part of the holonomy which is termed translational holonomy. In this case, the loop variable is given by an expression similiar to the one given in eq. (2), with the Riemann tensor interchanged by the torsion tensor ${ }^{19}$, that is,

$$
U_{T}(C)=P \exp \left(i \int_{D} T_{b c}^{a} d x^{b} d x^{c}\right) .
$$


The Lense-Thirring spacetime doesn't present torsion, however, it presents a translational holonomy due to the angular momentum. In this situation we consider the development of the curve $C$, for example, on the equatorial plane, in this spacetime. The definition of the development of a curve, $C$, is the following ${ }^{20}$ :

Given a curve $C:[0,1] \rightarrow M$, the development of the curve on the flat manifold $M^{\prime}$ is the curve $C^{\prime}:[0,1] \rightarrow M^{\prime}$ defined by

$$
D_{u}^{\prime} u^{\prime}=A^{\prime}(s) L[(A)]^{-1} D_{u} u
$$

with $C^{\prime}(0)=P^{\prime}$ and $u^{\prime}(0)=L u(0)$, where $P \in M, P^{\prime} \in M^{\prime}$. Note that $A: T_{C(0)}(M) \rightarrow T_{C(s)}(M)$, is the parallel transport along the curve $C ; L$ $: T_{p} M \rightarrow T_{p^{\prime}} M^{\prime}$ is an isometry, with $u$ and $u^{\prime}$ being the tangent vectors to $C$ and $C^{\prime}$, respectively. The operators $D$ and $D^{\prime}$ are the covariant differentials in $M$ and $M^{\prime}$, respectively.

In this paper we will calculate the holonomy transformation corresponding to circles on the equatorial plane in the spacetime of Lense-Thirring, taking into account the contributions arising from the linear and translational holonomies. This will be done in Section 2. In Section 3, we add some concluding remarks.

\section{Holonomy Transformation in the Lense-Thirring Spacetime}

The Lense-Thirring spacetime can be considered as a first approximation of the Kerr spacetime, valid for small values of the angular moment per unit mass of the source. Thus, in this approximation, the metric for a rotating gravitational body, in the coordinate system $(t, r, \theta, \varphi)$, is given by ${ }^{21}$

$$
\begin{aligned}
d s^{2}= & -\frac{\Delta}{r^{2}} d t^{2}-\frac{4 m a}{r} \sin ^{2} \theta d t d \phi+\frac{r^{2}}{\Delta} d r^{2} \\
& +r^{2} d \theta^{2}+r^{2} \sin ^{2} \theta d \phi^{2},
\end{aligned}
$$

where $m$ and $a$ are the mass and angular momentum per unit mass, respectively. Note that we assumed $\Delta \approx r^{2}-2 m r$, which means that we have neglected the term containing the second power of the angular momentum per unit mass of the source as compared with the definition of this parameter in Kerr spacetime.

In order to calculate the holonomy transformation we should find the expressions for the tetradic connections. Then, let us introduce a set of four vectors $e_{(a)}^{\mu}($ the Greek letter $\mu=0,1,2,3$ represents a Lorentz index and the Latin letter $a=0,1,2,3$ is the tetradic index), which are orthonormal at each point with respect to the metric with Minkowski signature, that is, $g_{\mu \nu} e_{(a)}^{\mu} e_{(b)}^{\nu}=\eta_{a b}=\operatorname{diag}(-1,+1,+1,+1)$. We will assume that the matrices $e_{(a)}^{\mu}$ have inverse $e_{\mu}^{(a)}$, such that $e_{\mu}^{(a)} e_{(a)}^{\nu}=\delta_{\mu}^{\nu}$ and $e_{\mu}^{(a)} e_{(b)}^{\mu}=\delta_{b}^{a}$. Considering the one-forms $\omega^{a}$ as

$$
\begin{aligned}
& \omega^{0}=\Delta^{1 / 2} r^{-1} d t-a \Delta^{1 / 2} r^{-1} \sin ^{2} \theta d \phi, \\
& \omega^{1}=\Delta^{-1 / 2} r d r, \\
& \omega^{2}=r d \theta, \\
& \left.\omega^{3}=-a r^{-1} \sin \theta d t++r \sin \theta\right) d \phi,
\end{aligned}
$$


we obtain, in the coordinate system $\left(x^{0}=t, x^{1}=r, x^{2}=\theta\right.$ e $\left.x^{3}=\phi\right)$, the tetrad frame defined by $\omega^{a}=e_{\mu}^{(a)} d x^{\mu}$, as

$$
\begin{aligned}
e_{0}^{(0)} & =\Delta^{1 / 2} r^{-1}, & e_{2}^{(2)} & =r \\
e_{3}^{(0)} & =-a \Delta^{1 / 2} r^{-1} \sin ^{2} \theta, & e_{0}^{(3)} & =-a r^{-1} \sin \theta, \\
e_{1}^{(1)} & =\Delta^{-1 / 2} r, & e_{3}^{(3)} & =-a r^{-1} \sin ^{2} \theta+r \sin \theta .
\end{aligned}
$$

Using Cartan's structure equation $d \omega^{a}=e_{\mu \| \nu}^{a} d x^{\nu} \wedge d x^{\mu}=-\omega_{b}^{a} \wedge \omega^{b}$, we obtain the following expression for the tetradic connections

$$
\begin{aligned}
\Gamma_{\mu 1}^{0} d x^{\mu} & =m r^{-2} d t \\
& +(m+r) r^{-2} a \sin ^{2} \theta d \phi=\Gamma_{\mu 0}^{1} d x^{\mu}, \\
\Gamma_{\mu 2}^{0} d x^{\mu} & =-a r^{-2} \Delta^{1 / 2} \cos \theta \sin \theta d \phi=\Gamma_{\mu 0}^{2} d x^{\mu}, \\
\Gamma_{\mu 3}^{0} d x^{\mu} & =-a \Delta^{-1 / 2} r^{-1} \sin \theta d r+a \Delta^{1 / 2} r^{-2} \cos \theta d \theta=\Gamma_{\mu 0}^{3} d x^{\mu}, \\
\Gamma_{\mu 2}^{1} d x^{\mu} & =-\Delta^{1 / 2} r_{-1} d \theta=-\Gamma_{\mu 1}^{2} d x^{\mu}, \\
\Gamma_{\mu 3}^{1} d x^{\mu} & =-\Delta^{1 / 2} r^{-1} \sin \theta d \phi=-\Gamma_{\mu 1}^{3} d x^{\mu}, \\
\Gamma_{\mu 3}^{2} d x^{\mu} & =2 m a r^{-3} \cos \theta d t- \\
& \cos \theta d \phi=-\Gamma_{\mu 2}^{3} d x^{\mu} .
\end{aligned}
$$

Firstly, let us calculate the linear holonomy, given by eq.(2), in the case where the curve is a circle with center at the origin with fixed values of $r, \theta$ and $t$. Then, we have

$$
\Gamma_{\mu} d x^{\mu}=\Gamma_{\phi} d \phi
$$

with $\Gamma_{\phi}$ given by

$$
\Gamma_{\phi}=\left(\begin{array}{cccc}
0 & A & B & 0 \\
A & 0 & 0 & C \\
B & 0 & 0 & D \\
0 & -C & -D & 0
\end{array}\right)
$$

where

$$
\begin{aligned}
& A=-a r^{-2}(m+r) \sin ^{2} \theta, \\
& B=-a \Delta^{1 / 2} r^{-1} \cos \theta \sin \theta, \\
& C=r^{-1} \Delta^{1 / 2} \sin \theta, \\
& D=\cos \theta .
\end{aligned}
$$

As $\Gamma_{\phi}$ does not depend of $\phi$, then, from eq. (2) we obtain

$$
U_{L}(c)=P \exp \left[\int_{\phi_{1}}^{\phi_{2}} \Gamma_{\phi} d \phi\right]=\exp \left[\Gamma_{\phi}\left(\phi_{2}-\phi_{1}\right)\right] .
$$

In particular, for the equatorial circle $(\theta=\pi / 2)$, we have

$$
\Gamma_{\phi}=\left(\begin{array}{cccc}
0 & A & 0 & 0 \\
A & 0 & 0 & C \\
0 & 0 & 0 & 0 \\
0 & -C & 0 & 0
\end{array}\right)
$$


where

$$
A=-\frac{a}{r}(1+m / r) \text { and } C=\frac{\Delta^{1 / 2}}{r}
$$

Notice that for $a=0$ and $r=2 m$, we have $\Gamma_{\phi}=0$ and therefore $U_{L}$ is equal to the unit matrix. From eq.(12) we have the following property

$$
\left(\Gamma_{\phi}\right)^{3}=-r^{-2} \Delta \Gamma_{\phi} \equiv-A_{\phi}^{2} \Gamma_{\phi},
$$

which implies that

$$
\begin{aligned}
U_{L} & =\exp \left[\Gamma_{\phi}\left(\phi_{2}-\phi_{1}\right)\right] \\
& =I+\frac{\Gamma_{\phi}}{A_{\phi}} \operatorname{sen}\left[A_{\phi}\left(\phi_{2}-\phi_{1}\right)\right]+\frac{\Gamma_{\phi}^{2}}{A_{\phi}^{2}}\left(1-\cos \left[A_{\phi}\left(\phi_{2}-\phi_{1}\right)\right]\right) .
\end{aligned}
$$

Thus, taking $\phi_{2}=2 \pi$ and $\phi_{1}=0$, the matrix form of the linear holonomy is given by

$$
U_{L}=\left(\begin{array}{cccc}
1+\frac{A^{2}}{A_{\phi}^{2}}(1-\xi) & \frac{A}{A_{\phi}} \zeta & 0 & -\frac{A C}{A_{\phi}^{2}}(1-\xi) \\
\frac{A}{A_{\phi}} \zeta & \xi & 0 & \frac{C}{A_{\phi}} \zeta \\
0 & 0 & 1 & 0 \\
-\frac{A C}{A_{\phi}^{2}}(1-\xi) & -\frac{C}{A_{\phi}} \zeta & 0 & 1-\frac{C^{2}}{A_{\phi}^{2}}(1-\xi)
\end{array}\right)
$$

where $\xi=\cos \left(2 \pi A_{\phi}\right)$ and $\zeta=\sin \left(2 \pi A_{\phi}\right)$.

Now, let us calculate the translational holonomy, considering a curve contained in the equatorial plane. Denoting this curve by $C(s)=(0, r, \pi / 2,2 \pi s)$ for $s \in[0,1]$, we have that the development of $C$ in the flat space-time $M^{\prime}$ in the coordinates $\left(t^{\prime}, x^{\prime}, y^{\prime}, z^{\prime}\right)$, beginning at the point $P^{\prime}=\left(t_{0}^{\prime}, x_{0}^{\prime}, y_{0}^{\prime}, z_{0}^{\prime}\right)$, is given by ${ }^{20}$

$$
C^{\prime}(s)=\left[t^{\prime}(s), x^{\prime}(s), y^{\prime}(s), z^{\prime}(s)\right],
$$

where $t^{\prime}(s), x^{\prime}(s), y^{\prime}(s)$ and $z^{\prime}(s)$ are expressed as

$$
\begin{aligned}
t^{\prime}(s) & =k_{1} \cdot s+t_{0}^{\prime}, \\
x^{\prime}(s) & =k_{2} \cdot[\cos (2 \pi v s)-1]+x_{0}^{\prime}, \\
y^{\prime}(s) & =k_{2} \cdot \sin (2 \pi v s)+y_{0}^{\prime}, \\
z^{\prime}(s) & =z_{0}^{\prime},
\end{aligned}
$$

with $k_{1}$ and $k_{2}$ being given by

$$
\begin{aligned}
& k_{1}=\frac{4 \pi m a}{\Delta^{1 / 2}}, \\
& k_{2}=\frac{r^{2}}{\Delta^{1 / 2}} .
\end{aligned}
$$


Therefore, the translational holonomy ${ }^{20}$ associated with the curve $C$ is

$$
U_{T}(C)=C^{\prime}(1)-C^{\prime}(0)=k_{1},
$$

and thus depends explicitly on the rotation as we can see from eq. (22) in which $k_{1}$ is defined. If we consider the result given by eq.(24) and take the limit $1 / r^{2} \rightarrow 0$, we obtain that $U_{T}(C)=6 \pi \frac{m a}{r}$, which means that the translational holonomy depends linearly on the angular momentum of the source, in this limit.

Thus, the total holonomy $U=U_{L}+U_{T}$, for circles in the equatorial plane, which is the sum of the linear and translational holonomies for these curves, is given by

$$
U(C)=\left(\begin{array}{cccc}
1 & \mathbb{F} \zeta & 0 & -\mathbb{F}(1-\xi) \\
\mathbb{F} \zeta & \xi & 0 & -\zeta \\
0 & 0 & 1 & 0 \\
\mathbb{F}(1-\xi) & \zeta & 0 & \xi
\end{array}\right)
$$

where $\mathbb{F}=a[1+m / r]\left(r^{2}-2 m r\right)^{-1 / 2}$.

Therefore, in addition to the linear holonomy which contains a contribution due to the rotation of the source, there is also a contribution arising from the rotation which appears in the translational holonomy.

\section{Concluding Remarks}

These results show us explicitly the dependence of the holonomy transformation on the curve and also on the parameters $m$ and $a$, which defines the Lense-Thirring metric. In the specific case of the rotation, the result for a curve on the equatorial plane shows how the total holonomy is modified by this quantity, through the explicit dependence of both the linear and translational holonomy with the rotation.

One of the physical motivations to calculate the holonomy transformation is that it can help us to investigate the gravitomagnetic and the gravitational AharonovBohm effects which occur at classical level, in the framework of the general relativity theory. In fact, both effects are connected with the concept of holonomy, with the gravitomagnetism being considered as an analog of the gravitational AharonovBohm effect.

\section{Acknowledgments}

We acknowledge Conselho Nacional de Desenvolvimento Científico e Tecnológico (CNPq)-Brazil for partial financial support.

\section{References}

1. S. Mandelstam, Ann. Phys.(NY) 19, 1 (1962); Phys. Rev. 175, 1580 (1968).

2. T. T. Wu and C. N. Yang, Phys. Rev. D 12, 3845 (1975).

3. S. Mandelstam, Ann. Phys.(NY) 19, 25 (1962); Phys. Rev. 175, 1604 (1968).

4. C. G. Bollini, J. J. Giambiagi and J. Tiomno, Lett. Nuovo Cim. 31, 13 (1981). 
5. V. B. Bezerra and P. S. Letelier, Class. Quantum Grav. 8, L73 (1991); J. Math Phys. 37, 6271 (1996).

6. V. B. Bezerra, Braz. J. Phys. 22, 328 (1992).

7. Tony Rothman, George F. R. Ellis and J. Murugan, Class. Quantum Grav. 18, 1217 (2001).

8. D. Bini, R. T. Jantzen and B. Mashhoon, Class. Quantum Grav. 18, 1 (2001).

9. R. Maartens, B. Mashhoon and D. Matravers, Class. Quantum Grav. 19, 195 (2002).

10. D. Bini, R. T. Jantzen and B. Mashhoon, Class. Quantum Grav. 19, 17 (2002).

11. D. Bini, C. Cherubini and R. T. Jantzen, Class. Quantum Grav. 19, 5481 (2002).

12. A. M. de M. Carvalho, F. Moraes and C. Furtado, Class. Quantum Grav. 20, 2063 (2003).

13. J. G. de Assis, C. Furtado, V. B. Bezerra and F. Moraes, Grav. Cosm. 6, pagina (2000).

14. A. M. de M. Carvalho and C. Furtado, Gen. Rel. Grav. 39, 1311 (2007).

15. D. Shao, L. Shao, H. Noda and C. G. Shao, Int. J. Mod. Phys. 19, 5247 (2004).

16. A. Barros, V. B. Bezerra and C. Romero, Mod. Phys. Lett. 18, 2673 (2003).

17. J. S. Dowker, Nuovo Cim. B 52, 129 (1967); L. H. Ford and A. Vilenkin, J. Phys. A 14, 2353 (1981).

18. V. B. Bezerra, Ann. Phys. (NY), 203, 392 (1990).

19. K. P. Tod, Class. Quantum Grav. 11, 1331 (1994).

20. R. J. Petti, Gen. Rel. Grav. 18, 5 (1986).

21. E. T. Newman, R. Couch, K. Chinnapared, A. Exton, A. Prakash and R. Torrence, J. Math. Phys. 6, 918 (1965). 